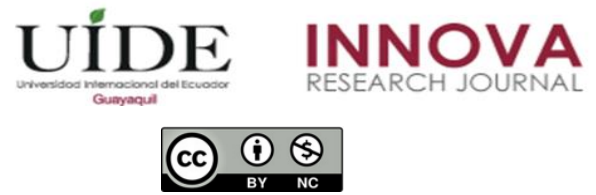

INNOVA Research Journal, ISSN 2477-9024

(Mayo-Agosto 2021). Vol. 6, No.2 pp. 225-239

DOI: https://doi.org/10.33890/innova.v6.n2.2021.1700

URL: http://revistas.uide.edu.ec/index.php/innova/index

Correo: innova@uide.edu.ec

\title{
Indicadores financieros y rentabilidad en bancos grandes y medianos ecuatorianos, periodo 2016-2019
}

\section{Financial Factors and Profitability in the Large and Medium Ecuadorian Banks, period 2016-2019}

Gabriela Alejandra Calahorrano Arias

Universidad de Las Américas, Ecuador

gabriela.calahorrano@udla.edu.ec

https://orcid.org/0000-0002-4811-7625

Francisco Andrés Chacón Guerrero

Universidad de Las Américas, Ecuador

francisco.chacon@udla.edu.ec

(D) https://orcid.org/0000-0001-5619-3241

Universidad de Las Américas, Ecuador

Ana Belén Tulcanaza-Prieto

Universidad de Las Américas e Instituto de Altos Estudios Nacionales, Ecuador

ana.tulcanaza@udla.edu.ec; ana.tulcanaza@iaen.edu.ec

https://orcid.org/0000-0002-9201-6848

Recepción: 29/01/2021 | Aceptación: 09/03/2021 | Publicación: 10/05/2021

Cómo citar (APA, séptima edición):

Calahorrano Arias, G. A., Chacón Guerrero, F. A. y Tulcanaza-Prieto, A. B. (2021). Indicadores financieros y rentabilidad en bancos grandes y medianos ecuatorianos, periodo 2016-2019. Innova Research Journal, 6(2), 225-239. https://doi.org/10.33890/innova.v6.n2.2021.1700

\section{Resumen}

Este estudio examina el impacto de los indicadores financieros en la rentabilidad de los bancos privados ecuatorianos, entregando una metodología útil para la identificación de los factores internos relevantes que influyen en el rendimiento de la entidad y permiten así, que la toma de decisiones del corto y largo plazo sea más eficiente. La investigación realizada utilizó un modelo de regresión lineal múltiple basado en mínimos cuadrados ordinarios, analizando indicadores de liquidez, morosidad, calidad de activos, y apalancamiento como variables independientes, mientras que el rendimiento sobre activos (ROA) y el rendimiento sobre patrimonio (ROE) 
constituyeron las variables dependientes. El estudio utilizó 192 observaciones provenientes de las entidades bancarias: Banco de Guayaquil, Produbanco, Banco Internacional, y Banco Bolivariano durante el periodo 2016-2019. Los resultados de este estudio demuestran (1) una relación negativa significativa entre los indicadores de liquidez y morosidad con el rendimiento y (2) una relación positiva significativa entre la calidad de activos y apalancamiento con el ROA y ROE. Con estos resultados, se concluye que las variables independientes son relevantes en el rendimiento bancario, por lo que se las debe monitorear continuamente para su control efectivo. Este manuscrito, complementa a la literatura financiera al introducir un análisis estadístico del periodo 2016-2019, entregando una investigación previa a la ocurrencia de la pandemia Covid19, de manera que, se recomienda que estudios futuros realicen un análisis posterior a la emergencia sanitaria y se comparen dichos resultados para determinar los efectos en la rentabilidad de los bancos provocados por la pandemia.

Palabras claves: indicadores financieros; rentabilidad; liquidez; morosidad; calidad de activos; apalancamiento.

\begin{abstract}
This study examines the impact of financial indexes on the profitability of Ecuadorian private banks to contribute to the financial management of banking institutions by providing a useful methodology to identify the internal factors that influence the banks' performance, and thus, the decision making process would be more efficient in the short- and long-term. This research used a multiple linear regression based on the econometric method of Ordinary Least Squares (OLS). Liquidity, non-performing loans, asset quality, and leverage are used as independent variables, while the return on assets (ROA) and the return on equity (ROE) are the dependent variables. The study used 192 observations of the following banking entities: Banco de Guayaquil, Produbanco, Banco Internacional, and Banco Bolivariano from 2016 to 2019. Our study shows (1) a significant negative relationship between liquidity and non-performing loans with the profitability, and (2) a significant positive relationship between the asset quality and leverage with ROA and ROE, which coincides with the findings of previous studies. To sum up, the selected independent variables are relevant to the bank performance, therefore, we suggest monitoring them continuously. This manuscript complements the financial literature by introducing a statistical analysis of the 20162019 period, providing prior research to the Covid19 pandemic. It is recommended that future studies carry out an analysis after the health emergency and compare the results of both studies to determine the effects on bank's profitability caused by the pandemic.
\end{abstract}

Keywords: financial indexes; profitability; liquidity; non-performing loans; asset quality; leverage.

\title{
Introducción
}

El propósito de este estudio es entregar una metodología que determine los factores internos relevantes en el rendimiento de la banca, esta información contribuirá a una gestión financiera más eficiente dentro de los bancos, ya que el entendimiento del impacto de los indicadores financieros en la rentabilidad permitirá que los directivos y la alta gerencia de las instituciones bancarias estén alertas a cualquier suceso que les afecte y así puedan tomar decisiones 
oportunas de corto y largo plazo que mitiguen el riesgo al que están expuestos y estimulen su crecimiento sostenible. Este documento científico se clasifica dentro de la literatura de finanzas y comercio debido a que su principal objetivo es apoyar al fortalecimiento de los actores de la economía y el desarrollo empresarial, en este caso este estudio influye en el afianzamiento de la rentabilidad de los bancos ecuatorianos.

De acuerdo con (Chavarin, 2015) un sistema financiero eficiente de banca transparente apoya al funcionamiento y desarrollo de las empresas, así como a la creación de nuevos negocios, de esta forma puede contribuir al crecimiento y desarrollo económico de un país. Sin embargo, su administración no siempre es la adecuada; por ejemplo, en Ecuador en 1999 el 70\% de las instituciones financieras quebraron por la crisis del feriado bancario (Redacción de Negocios, 2010). Esta crisis bancaria se produjo por la falta de control de la Superintendencia de Bancos del Ecuador sobre el otorgamiento de préstamos vinculados (Mendoza, 2002). Este hecho fue determinante para que se tomen medidas de control sobre la eficiencia y la administración de los bancos, con el fin de prevenir nuevas crisis financieras (Buenaño, 2003). A pesar de que los controles sobre la banca aumentaron en el periodo del 2000 al 2003, en esta época se mantenía la percepción de que la banca se administraba de manera ineficiente.

Luego de varios años de la implementación de distintas leyes y normas enfocadas en mejorar el control y la eficiencia de las instituciones bancarias ecuatorianas, se evidencia que, el sistema financiero ecuatoriano ha demostrado estabilidad y solidez, ya que ha logrado superar eventos desafiantes que han puesto a prueba su desempeño y sostenibilidad en la última década, lo que ha permitido que se ganen la confianza del público. (Asociación de Bancos Privados del Ecuador, 2014). Sin embargo, es importante mencionar que los bancos por su giro de negocio siempre están expuestos a varios riesgos que pueden afectar su desenvolvimiento como el riesgo de mercado, de crédito, de liquidez, operativo, entre otros. Por lo que las instituciones financieras deben monitorear permanentemente su rendimiento a través de indicadores financieros, permitiéndoles estar alerta y tomar decisiones oportunas sobre su gestión, y así mitigar los riesgos.

Antes de monitorear los indicadores financieros es fundamental entender el impacto de éstos sobre el rendimiento de la entidad bancaria, debido a que, al comprender la relación entre las variables, se facilita la toma de decisiones y la implementación de controles mitigantes de riesgo. En este sentido, este estudio busca contribuir con el análisis del impacto financiero de los indicadores de liquidez, morosidad, calidad de activos, y apalancamiento en la rentabilidad de los bancos ecuatorianos durante el periodo 2016-2019. El alcance de este estudio incluye a una muestra de bancos grandes y medianos de Ecuador, debido a que estudios previos analizan únicamente a los bancos ecuatorianos grandes.

Distintas investigaciones mencionan que los indicadores de liquidez y morosidad tienen una relación lineal negativa con el rendimiento. Por ejemplo, (Ricaurte y Rosero, 2017) y (Velasco, 2017) señalan que la relación entre la morosidad y la rentabilidad es inversa, es decir, que a medida que incrementa la morosidad se reduce el rendimiento, lo que conlleva el aumento del riesgo de liquidez y de crédito, si no se controlan a tiempo, podrían causar el cierre de un banco. Así mismo, (Torres, 2018) y (Moreno, 2019) establecen que a menor liquidez mayor rentabilidad, por lo que, indican que se requiere un nivel óptimo de liquidez que permita cumplir con las obligaciones financieras de la entidad bancaria, manteniendo su rentabilidad, es decir, optimizando el trade-off 
entre la liquidez y la rentabilidad.

Por otro lado, diversos estudios empíricos señalan que los indicadores de calidad de activos y apalancamiento tienen una relación lineal positiva con la rentabilidad. En particular, (Gutierrez y Garrido, 1994) y (Eslava, Chacón, y González, 2017) mencionan en sus investigaciones que la calidad de activos permite un crecimiento y mejora de la rentabilidad de los bancos analizados en América del Norte y Venezuela, respectivamente. Además, (Rodríguez y Venegas, 2012) y (Jara, Sánchez, Bucarám, y García, 2018) indican que los bancos que generan mayor rentabilidad tienen mayor apalancamiento de largo plazo, lo que permite concluir que el apalancamiento posee una relación positiva con el rendimiento de las instituciones financieras.

Ante esta problemática, la presente investigación analiza el efecto de los indicadores financieros de liquidez, morosidad, calidad de activos, y apalancamiento sobre el rendimiento de los bancos ecuatorianos medido a través del rendimiento sobre activos (ROA) y el rendimiento sobre patrimonio (ROE). El estudio tomó como muestra a dos bancos grandes (Banco de Guayaquil y Produbanco) y dos bancos medianos (Banco Internacional y Banco Bolivariano), cuyos niveles de activos en conjunto representan el $40 \%$ del total de activos del sistema financiero ecuatoriano, lo que demuestra la representatividad de la muestra, de la cual se obtuvieron 192 observaciones extraídas de los balances mensuales durante el periodo 2016-2019.

La investigación utiliza un modelo de regresión lineal múltiple que permite determinar la relación entre las variables independientes y la variable dependiente, además se comprueban las hipótesis planteadas en este estudio. Una vez corrido el modelo se demuestra que las variables independientes liquidez y morosidad tienen una relación lineal inversa significativa con el rendimiento, mientras que la calidad de activos y apalancamiento tienen una relación directa significativa con las variables dependientes ROA y ROE, siendo las cuatro variables independientes significativas al $1 \%$. Con estos resultados, se concluye que se puede incrementar la rentabilidad de las entidades bancarias si se aplican políticas adecuadas respecto a los niveles de liquidez, recuperación de cartera, otorgamiento de cartera, y niveles de apalancamiento.

El artículo se compone de cuatro secciones. El segundo apartado muestra la revisión de la literatura que refiere al marco teórico y definición de las hipótesis. La tercera sección muestra el modelo de investigación. La cuarta sección describe el estudio empírico y resultados, mientras que el último apartado señala las conclusiones y las recomendaciones.

\section{Marco teórico}

Las entidades financieras cumplen un rol relevante en la economía de un país o nación, puesto que su actividad principal es la intermediación financiera, es decir, otorgan créditos de los recursos procedentes de los depósitos que reciben, permitiendo relocalizar los fondos de los agentes superavitarios a los agentes deficitarios de la economía. Sin embargo, esta actividad está expuesta a varios riesgos que, si no son administrados de forma correcta, pueden afectar su estabilidad y la del sistema financiero. Por lo que, varios organismos internacionales como: el Comité de Basilea para la Supervisión Bancaria, el Banco Mundial y el Fondo Monetario 
Internacional buscan maneras de fortalecer la estabilidad financiera en todo el mundo, una muestra es la emisión de los Principios Básicos para la Supervisión Bancaria de Basilea (Comité de Basilea, 1997).

La implementación de los principios de Basilea y las normas y leyes emitidas por la Junta de Regulación Monetaria Financiera del Ecuador, ha permitido que el sistema financiero ecuatoriano sea sólido y estable, el mismo que se ha puesto a prueba en varios momentos (Tenesaca, Villanueva, Malo, y Higuerey, 2017). Siendo, por ejemplo, algunos episodios difíciles para la economía ecuatoriana los siguientes: (1) año 2015, reducción del precio del barril de petróleo que provocó disminución en los ingresos del Estado, (2) año 2019, implementación de medidas económicas como la eliminación del subsidio a los hidrocarburos para reducir los gastos del Estado y (3) año 2020, efectos económicos y sociales ocasionados por la emergencia sanitaria por la pandemia Covid19.

Es por ello que, una manera para asegurar la estabilidad de los bancos y mitigar los riesgos a los que están expuestos por su giro de negocio es el monitoreo continuo de los indicadores financieros y el impacto que ejercen sobre su rentabilidad. Según (Rodrigues, 2015) la rentabilidad se define como el rendimiento que un agente económico espera percibir de la operación de una inversión, se mide por la proporción entre el resultado de un periodo y el valor de la inversión. Además, la rentabilidad puede ser determinada por factores internos de las entidades bancarias como los indicadores financieros tales como: liquidez, morosidad, calidad de activos $\mathrm{y}$ apalancamiento, estos permiten tener un panorama general del desempeño de la entidad financiera y pueden ser determinantes significativos de la rentabilidad, de esta premisa se despliegan las hipótesis de este estudio.

La rentabilidad puede ser medida a través de varios indicadores como el ROA que relaciona al rendimiento con el capital invertido, mientras que el ROE representa la rentabilidad generada para los accionistas, es decir, está relacionada con la creación de valor para los inversionistas. En múltiples investigaciones realizadas en varios países, generalmente se utiliza como variables dependientes en sus modelos tanto al ROA como al ROE para establecer los determinantes de la rentabilidad (Rodrigues, 2015).

La liquidez es la capacidad de los activos para transformarse en efectivo en poco tiempo de forma fácil y rápida. (BBVA, 2021) En cuanto a los indicadores de liquidez, estos permiten medir el nivel de fondos disponibles de una entidad para poder cumplir con sus pasivos a corto plazo (Asociación de Bancos Privados del Ecuador, 2020). La importancia de la liquidez radica en brindar seguridad a los depositantes de que el banco no incurra en riesgo de liquidez. Según la (Superintendencia de Bancos, 2004) el riesgo de liquidez se define como la posibilidad de pérdida por la incapacidad de la entidad financiera para afrontar escasez de fondos y cumplir con sus obligaciones. Es importante mencionar que existe un trade-off entre la liquidez y la rentabilidad, ya que, tener activos líquidos no genera ganancias a diferencia de los activos productivos como cartera, por lo tanto, el costo de oportunidad de los recursos líquidos es alto. Es por ello que, es sustancial monitorear tanto los indicadores de liquidez como los indicadores de rentabilidad para poder establecer un nivel óptimo de liquidez que permita cumplir con las obligaciones y maximizar la rentabilidad obtenida. 
Refiriéndose a estudios empíricos previos, la investigación (Torres, 2018) demuestra que las instituciones bancarias pueden cumplir con sus obligaciones financieras, manteniendo una estabilidad económica y financiera sin afectar su rentabilidad. Otro aporte de (Moreno, 2019) señala que la liquidez incide negativamente de forma directa en la rentabilidad tanto de los activos como del patrimonio de los bancos ecuatorianos, pues a menor liquidez mayor rentabilidad en la banca privada de Ecuador. Esta información concuerda con lo que se va a demostrar en este estudio.

Hipótesis 1: El índice de liquidez afecta negativamente a la rentabilidad de las instituciones financieras.

Por otro lado, es importante mencionar que la cartera es el activo principal de los bancos, pues genera ingresos por los intereses que se cobra a los prestatarios, por lo que es fundamental que se analicen los indicadores relacionados a los activos. Si no se realiza un análisis adecuado o se realiza un seguimiento deficiente a la cartera, la entidad bancaria puede incurrir en riesgo de crédito. La (Superintendencia de Bancos, 2004) define al riesgo de crédito como la probabilidad de pérdida debido al incumplimiento del prestatario por el no pago de las obligaciones pactadas con la institución financiera. Cabe señalar que existen varios indicadores referentes a los activos, tales como: morosidad, cobertura de cartera improductiva, calidad de activos, entre otros, que permiten controlar el riesgo de crédito.

Según (Díaz, 2018) la morosidad se define como la incapacidad de los agentes económicos para pagar sus deudas en el tiempo previsto, además la morosidad es la relación entre la cartera improductiva y la cartera bruta, de forma que este índice se vincula con la recuperación de los créditos. Cabe mencionar que, en los momentos en los que se ha puesto a prueba la estabilidad de las instituciones financieras, el nivel de morosidad de los bancos tiende a crecer considerablemente, limitando los recursos disponibles hacia nuevos prestatarios, esto a su vez, influye en el rendimiento, pues no se producen nuevos ingresos y no se recupera el capital prestado ni los intereses.

En estudios previos como en la investigación de (Ricaurte y Rosero, 2017) indican que un alto índice de morosidad conlleva un riesgo de liquidez e incluso podría causar el cierre de instituciones financieras por su afectación negativa en su rentabilidad. Por otro lado, la investigación de (Chavarin, 2015) señala que el índice de morosidad no resulta un factor determinante de la rentabilidad de la banca comercial en México, lo que contrapone lo expuesto por (Velasco, 2017) en su investigación que confirma la relación inversa de la morosidad y la rentabilidad en los bancos privados grandes de Ecuador, sin embargo, menciona que la afectación no es significativa.

Hipótesis 2: La morosidad de cartera afecta negativamente a la rentabilidad de las instituciones financieras.

Según (Asociación de Bancos Privados del Ecuador, 2020) la calidad de activos es un indicador de eficiencia que mide la relación entre los activos que producen intereses (cartera) y los pasivos que generan costos (depósitos a la vista y a plazo). En otras palabras, la calidad de activos 
es el cociente entre los activos productivos y los pasivos con costo. Este indicador muestra el nivel de eficiencia de la colocación de los recursos captados. Si la calidad de activos, se administra adecuadamente puede generar mayor rendimiento para la institución financiera, esto implica que los recursos de captaciones están siendo colocados eficientemente en operaciones de crédito productivas, que no presentan problemas en su recuperación.

De acuerdo con la investigación de (Gutierrez y Garrido, 1994) en la cual analiza la calidad de activos de la banca comercial de Canadá, Estados Unidos, y México, se determina que en el periodo estudiado existió crecimiento de la utilidad neta por un incremento de los activos productivos. Otro aporte de (Eslava, Chacón, y González, 2017) señala que la calidad de activos de los veinte y tres bancos analizados de Venezuela tiene proporciones adecuadas, lo que mejora su rentabilidad y se demuestra que la calidad de activos tiene una influencia positiva en la rentabilidad de los bancos.

Hipótesis 3: La calidad de activos afecta positivamente a la rentabilidad de las instituciones financieras.

Según (Asociación de Bancos Privados del Ecuador, 2018) el apalancamiento financiero busca determinar la proporción del endeudamiento de los bancos que es financiado por el patrimonio, de modo que el apalancamiento se calcula como la razón del pasivo sobre el patrimonio. Es importante monitorear al apalancamiento, ya que un nivel muy alto de apalancamiento puede provocar inestabilidad en el sistema financiero como por ejemplo en la crisis financiera internacional de 2008. Por el contrario, si se maneja de forma adecuada, el apalancamiento puede impulsar una mayor rentabilidad, permitiendo que la institución financiera tenga mayores recursos que invertir y éstos generen ganancias.

De acuerdo con los resultados de estudios previos, (Rodríguez y Venegas, 2012) muestran que los bancos de mayor tamaño en México, que generan mayor rentabilidad presentan mayor apalancamiento de largo plazo. Así mismo, (Jara, Sánchez, Bucarám, y García, 2018) obtuvieron como resultado que los depósitos a plazo, la cartera bruta, el apalancamiento, y la intermediación financiera tienen mayor influencia en la rentabilidad de los bancos pequeños de Ecuador. En particular, el apalancamiento tiene una relación positiva significativa con el ROE, lo que coincide con lo que se va a demostrar en este estudio. financieras.

Hipótesis 4: El apalancamiento afecta positivamente a la rentabilidad de las instituciones

\section{Metodología}

El propósito de esta investigación es establecer el impacto de los indicadores financieros de liquidez, morosidad, calidad de activos, y apalancamiento en la rentabilidad de una muestra de cuatro bancos ecuatorianos que incluye dos bancos grandes (Banco Guayaquil, Produbanco) y dos bancos medianos (Banco Internacional y Banco Bolivariano) cuya trayectoria es mayor a 40 años en el mercado ecuatoriano. Se seleccionaron estos indicadores financieros porque existe evidencia en algunos estudios previos de países como México y Venezuela que demuestran la relación de las 
variables propuestas, por consiguiente, esta investigación busca aplicarlo en el contexto de Ecuador.

La información fue extraída del repositorio de balances generales mensuales del periodo de 2016 a 2019 de la Superintendencia de Bancos del Ecuador. Esta investigación utiliza una regresión lineal múltiple a través de Mínimos Cuadrados Ordinarios (MCO) como modelo para comprobar las hipótesis antes mencionadas, además este modelo refuerza el enfoque teórico y empírico a través de la siguiente ecuación:

\section{Ecuación 1}

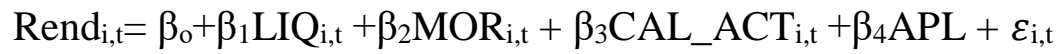

Donde:

Rend $_{i, t}=$ variable dependiente, medida a través de $\mathrm{ROA}_{\mathrm{i}, \mathrm{t}} \mathrm{y} \mathrm{ROE}_{\mathrm{i}, \mathrm{t}}$ para el banco $i$ en el mes $t$, $\beta_{\mathrm{o}}=$ intercepto,

$\varepsilon_{\mathrm{i}, \mathrm{t}}=$ representa el error para el banco $i$ en el mes $t$

Las variables que componen la ecuación se detallan a continuación (Tabla 1):

\section{Tabla 1}

\section{Variables del modelo}

\begin{tabular}{lll}
\hline Etiqueta & Variables & Fórmula de Cálculo \\
\hline \multicolumn{2}{l}{ Variables Dependientes } & \\
ROA & Retorno sobre los activos & (Ingresos - Gastos) / Activo \\
ROE & Retorno sobre el patrimonio & (Ingresos - Gastos) / Patrimonio \\
Variables Independientes & \\
LIQ & Liquidez & Fondos disponibles / Depósitos a corto plazo \\
MOR & Morosidad & Cartera Improductiva / Cartera bruta \\
CAL_ACT & Calidad de activos & Activos productivos / Pasivos con costo \\
APL & Apalancamiento & Pasivo total / Patrimonio total \\
\hline
\end{tabular}

Nota: Todas las variables están expresadas para el banco i en el mes t

Fuente: (Asociación de Bancos Privados del Ecuador, 2020)

Elaboración: Propia

Dentro de la metodología, se realizó un análisis de estadísticos descriptivos para verificar el comportamiento histórico de las variables. Posteriormente, se ejecutó un análisis de correlación, en el que se muestra que la asociación lineal entre las variables, para verificar que no exista multicolinealidad entre las mismas. Finalmente, se corrió la regresión de MCO verificando el cumplimiento de supuestos.

\section{Resultados}

La Superintendencia de Bancos utiliza la metodología de percentiles para clasificar a los bancos ecuatorianos por su tamaño según la participación de sus activos en el sector, de forma que, 
se consideran bancos grandes a las instituciones con una participación mayor al 36\%, bancos medianos a los que participan entre el $12 \%$ y $36 \%$ y bancos pequeños a los que poseen una participación menor al 12\%. (Jara, Sánchez, Bucarám, y García, 2018) La muestra de este estudio consideró a cuatro bancos privados, entre ellos, dos bancos grandes: Banco Guayaquil y Produbanco y dos bancos medianos: Banco Internacional y Banco Bolivariano. Se seleccionaron estos bancos porque los activos de estas entidades bancarias representan en conjunto el $40 \%$ del total de activos del sistema, así mismos el par de bancos grandes representan el $37 \%$ del total de activos de su categoría y el par de bancos medianos tienen una participación del $50 \%$ del total de activos de su categoría, demostrando la representatividad y significatividad de estos bancos en el sistema financiero ecuatoriano. (Asociación de Bancos Privados del Ecuador, 2020). Además, este estudio buscaba una muestra hibrida de bancos grandes y medianos a diferencia de estudios previos que consideran bancos de una sola categoría grandes, medianos o pequeños.

La información requerida para calcular los indicadores financieros se obtuvo de los balances generales mensuales de estas instituciones financieras, misma que se extrajo del repositorio de la Superintendencia de Bancos. Inicialmente el estudio analizaba 232 observaciones del periodo comprendido entre enero 2016 a octubre 2020. Sin embargo, las observaciones de abril 2020 a octubre 2020 mostraban datos atípicos explicados por la crisis económica, social, y sanitaria producida por la pandemia Covid19. De manera que se decidió excluir al año 2020 de la investigación obteniendo como muestra final 192 observaciones (Tabla 2).

\section{Tabla 2}

\section{Selección de la muestra}

\begin{tabular}{ll}
\hline Detalle & Número \\
\hline Muestra inicial de observaciones ene 2016 - oct 2020 & 232 \\
(-) Observaciones con valores atípicos ene 2020 - oct 2020 & 40 \\
(=) Muestra final observaciones ene 2016 - dic 2019 & 192 \\
\hline
\end{tabular}

\section{Estadística descriptiva}

Los datos obtenidos en el análisis de estadística descriptiva de los bancos seleccionados (Banco Guayaquil, Produbanco, Banco Internacional y Banco Bolivariano) se muestran en la Tabla 3. La media de las variables dependientes ROA y ROE es 0.0107 y 0.1163 , respectivamente. Como se visualiza la media del ROE es mayor a la del ROA, esto se debe a que de manera general el ROE tiene en su denominador al patrimonio mientras que el denominador del ROA es el activo, contablemente el activo es la suma del pasivo y el patrimonio, por lo que el denominador del ROE es menor al denominador del ROA. 
Gabriela Alejandra Calahorrano Arias, Francisco Andrés Chacón Guerrero y Ana Belén Tulcanaza-Prieto.

ISSN 2477-9024. Innova Research Journal (Mayo-Agosto, 2021). Vol 6, No. 2, pp. 225-239

\section{Tabla 3}

Estadísticas descriptivas

\begin{tabular}{lccccccc}
\hline Variables & \multicolumn{1}{c}{ Media } & $\begin{array}{c}\text { Desviación } \\
\text { standard }\end{array}$ & Mínimo & Cuartil 1 & Mediana & Cuartil 3 & Máximo \\
\hline Variables Dependientes & & & & & & & \\
ROA & 0,0107 & 0,0033 & 0,0039 & 0,0076 & 0,0109 & 0,0131 & 0,0194 \\
ROE & 0,1163 & 0,0346 & 0,0454 & 0,0871 & 0,1198 & 0,1465 & 0,1762 \\
Variables Independientes & & & & & & & \\
LIQ & 0,3009 & 0,0557 & 0,1916 & 0,2571 & 0,3000 & 0,3394 & 0,4301 \\
MOR & 0,0192 & 0,0077 & 0,0087 & 0,0130 & 0,0167 & 0,0231 & 0,0385 \\
CAL_ACT & 1,2762 & 0,1528 & 0,9751 & 1,1475 & 1,2886 & 1,4081 & 1,5421 \\
APL & 0,0913 & 0,0094 & 0,0782 & 0,0839 & 0,0881 & 0,0961 & 0,1153 \\
\hline
\end{tabular}

Nota: Las variables dependientes son (1) Rendimiento sobre los activos (ROA), (2) Rendimiento sobre el patrimonio (ROE) y las variables independientes son (1) Liquidez (LIQ), (2) Morosidad (MOR), (3) Calidad de Activos (CAL_ACT) y (4) Apalancamiento (APL). N= 192.

\section{Análisis de correlación}

La tabla 4 muestra la matriz de correlación de Pearson. Se observa que las variables independientes liquidez, morosidad, calidad de activos, y apalancamiento son significativas al nivel de $1 \%$ respecto al ROA, con coeficientes de $-0.3706,-0.5093,-0.2507$ y 0.2376 , respectivamente, mientras que, para el ROE, las variables independientes son significativas al nivel de $1 \%$ y $10 \%$, a excepción del apalancamiento, con coeficientes de $-0.4559,-0.6219,-0.1711$ y 0.0423, respectivamente. La asociación lineal de la liquidez, la morosidad, y la calidad de activos es negativa con el ROA y ROE, mientras que el apalancamiento está correlacionado positivamente con el ROA y negativamente con el ROE. También, se observa que los valores de correlación entre las variables independientes no superan a 0,7000 , por lo que la multicolinealidad no se incrementa dado que los valores absolutos se ubican en el rango de 0,0369 y 0,6219, identificándose de esta forma la no prevalencia del problema de colinealidad entre las variables (Gurajati, 1988).

\section{Tabla 4}

Matriz de correlación de Pearson

\begin{tabular}{|c|c|c|c|c|c|c|}
\hline & ROA & ROE & LIQ & MOR & CAL_ACT & APL \\
\hline ROA & 1 & & & & & \\
\hline ROE & $0,9399 * * *$ & 1 & & & & \\
\hline LIQ & $-0,3706^{* * *}$ & $-0,4559 * * *$ & 1 & & & \\
\hline MOR & $-0,5093 * * *$ & $-0,6219 * * *$ & 0,1072 & 1 & & \\
\hline CAL ACT & $-0,2507 * * *$ & $-0,1711 *$ & 0,0369 & $0,2803 * * *$ & 1 & \\
\hline APL & $0,2376 * * *$ & $-0,0423$ & $0,2485 * * *$ & $0,3102 * * *$ & $-0,2931$ & 1 \\
\hline
\end{tabular}

Nota: *** y * indican significancia estadística al $1 \%$ y al $10 \%$, respectivamente. 


\section{Análisis de regresión lineal}

La tabla 5 muestra los resultados de las dos regresiones lineales múltiples explicando el comportamiento del ROE en la primera regresión y del ROA en la segunda regresión, en función de sus predictores que son los indicadores de liquidez, morosidad, calidad de activos, y apalancamiento. Es importante mencionar que el $\mathrm{R}$ cuadrado muestra que las variables independientes explican en un $62.57 \%$ a la variable dependiente ROE y en un $62.10 \%$ al ROA.

Los resultados de los modelos del ROE y ROA muestran que las variables independientes de liquidez y morosidad tienen una relación lineal inversa significativa con las variables dependientes expresada con el signo negativo en los coeficientes obtenidos, mientras que las variables independientes de calidad de activos y apalancamiento tienen una relación lineal directa significativa con las variables dependientes ROE y ROA expresada con el signo positivo en los coeficientes obtenidos. Los coeficientes indican que cuando una de las variables independientes: liquidez, morosidad, calidad de activos o apalancamiento cambia en una unidad y el resto de las variables independientes se mantienen constantes, la variable dependiente ROE disminuye o aumenta en: $-0.2911,-3.2152,0.0334$, y 1.2466 , respectivamente y la variable dependiente ROA disminuye o aumenta en: $-0.0271,-0.2961,0.0029$, y 0.2128, respectivamente. (Tabla 5).

En los dos modelos se concluye que la relación entre las variables independientes y el ROE y ROA es significativa. Los modelos de regresión lineal múltiple muestran que todas las variables independientes (liquidez, morosidad, calidad de activos, y apalancamiento) son significativas al $1 \%$, implicando que, los resultados son confiables y permiten aceptar las hipótesis planteadas al inicio de este estudio que señalan que los indicadores financieros de liquidez y morosidad afectan negativa y significativamente al rendimiento de las instituciones financieras ecuatorianas mientras que la calidad de activos y el apalancamiento afectan positiva y significativamente a la rentabilidad de los bancos en el Ecuador.

\section{Tabla 5}

\section{Resultados regresión}

\begin{tabular}{lll}
\hline & ROE & ROA \\
\hline Constante & $0,1093^{* * *}$ & 0,0014 \\
& $(4,2863)$ & $(0,5688)$ \\
LIQ & $-0,2911^{* * *}$ & $-0,0271^{* * *}$ \\
& $(-10,0154)$ & $(-9,7090)$ \\
MOR & $-3,2152 * * *$ & $-0,2961 * * *$ \\
& $(-13,8463)$ & $(-13,2885)$ \\
CAL_ACT & $0,0334 * *$ & $0,0029 * * *$ \\
& $(2,8373)$ & $(2,6044)$ \\
APL & $1,2466 * * *$ & $0,2128 * *$ \\
& $(6,3189)$ & $(11,2443)$ \\
$\mathrm{R}^{2}$ & 0,6257 & 0,6210 \\
$\mathrm{R}^{2}$ ajustado & 0,6177 & 0,6129 \\
Estadístico F & 76,5903 & 78,1443 \\
$\mathrm{~N}$ & 192 & 192 \\
\hline
\end{tabular}

Nota: Beta corresponde a los coeficientes no estandarizados, los números en paréntesis son el valor $\mathrm{t}$ estadístico, *** 
indica la significancia estadística al 1\%

Los resultados de los modelos muestran que el impacto de la liquidez en el ROE y en el ROA es negativo significativamente al $1 \%$. Estos hallazgos concuerdan con los resultados del estudio de (Moreno, 2019) que muestran que la liquidez incide de forma directa negativa en la rentabilidad tanto de los activos como del patrimonio de los bancos en Ecuador. Es importante mencionar que las instituciones financieras requieren establecer niveles mínimos de liquidez para no incurrir en riesgo de liquidez, aunque esto implica renunciar a un mayor porcentaje de rendimiento como se demuestra en esta investigación, ya que si aumenta la liquidez disminuye la rentabilidad. Por lo tanto, se requiere que se realice un monitoreo permanente de los niveles de liquidez para que se encuentre un nivel óptimo que minimice el riesgo de liquidez y maximice el rendimiento.

Así mismo, en ambos modelos se observa que la morosidad tiene una relación inversa significativa con el rendimiento, esto demuestra que el incumplimiento de obligaciones de los prestatarios con las entidades financieras tiene un impacto negativo relevante en el rendimiento de la banca, sin embargo, esto se contrapone a lo mencionado por (Chavarin, 2015) quien señala que el índice de morosidad no resulta un factor determinante de la rentabilidad de la banca comercial en México. Por otro lado, (Cabellos y Naito, 2015) confirman los hallazgos de esta investigación y explican que una mayor morosidad genera una menor rentabilidad. De tal manera, se determina que es necesario profundizar el análisis de riesgo de crédito, prevenir moras, recuperar cartera, optimizar el seguimiento, monitoreo y evaluación de la morosidad para mejorar la rentabilidad (Heredia y Torres, 2019).

Además, los dos modelos tanto del ROE como el ROA, indican que la calidad de activos tiene influencia positiva en la rentabilidad. Estos hallazgos coindicen con los resultados del estudio de (Gutierrez y Garrido, 1994) sobre la calidad de activos de la banca comercial del bloque de América del Norte y con los resultados de la investigación de (Eslava, Chacón, y González, 2017) sobre los indicadores financieros y la banca universal de Venezuela. Ambos estudios establecen que a mayor calidad de activos hay una mejora y crecimiento de la rentabilidad. Por consiguiente, se establece que los bancos deben realizar un exhaustivo análisis de crédito antes de colocar la cartera, ya que de esta forma garantizan que la calidad de los activos se mantenga en proporciones adecuadas para que favorezcan a su rendimiento y demuestren su eficiencia en la colocación de los recursos que captan.

Por último, los hallazgos de los modelos respecto al apalancamiento muestran que la relación de este indicador con el ROE y ROA es positiva y significativa, esto implica que a mayor apalancamiento mayor rentabilidad. Así mismo, los resultados del estudio de (Rodríguez y Venegas, 2012) sobre la liquidez y el apalancamiento en los bancos de México y los resultados de la investigación de (Jara, Sánchez, Bucarám, y García, 2018) sobre los indicadores de rentabilidad de los bancos pequeños de Ecuador después de la dolarización, señalan que los bancos que tienen mayor apalancamiento a largo plazo generan mayor rentabilidad. Por lo contrario, (Vargas y Díaz, 2017) exponen que un alto nivel de apalancamiento conlleva a inestabilidad, ya que el riesgo es mayor cuando la rentabilidad aumenta y se puede perder todo como en la crisis financiera internacional de 2008. De modo que los bancos deben establecer niveles prudentes de apalancamiento que les permitan crecer minimizando su riesgo. 


\section{Conclusiones y recomendaciones}

Este estudio analiza la relación entre los indicadores financieros y la rentabilidad en el entorno bancario ecuatoriano en el periodo 2016-2019, usando 192 observaciones obtenidas de los balances mensuales publicados en el repositorio de la Superintendencia de Bancos de Ecuador, utilizando una muestra de cuatro bancos privados, compuesta por dos bancos grandes tales como, Banco de Guayaquil y Produbanco, y dos bancos medianos, Banco Internacional y Banco Bolivariano. El modelo desarrollado es una regresión lineal múltiple a través de MCO, que considera como variables independientes a los indicadores de liquidez, morosidad, calidad de activos, y apalancamiento mientras que los indicadores de rentabilidad ROA y ROE son las variables dependientes.

Los hallazgos del modelo fueron que los indicadores de liquidez y morosidad tienen una relación inversa significativa con los indicadores de rendimiento ROA y ROE, mientras que los indicadores de calidad de activos y apalancamiento tienen una relación directa significativa con el rendimiento de las instituciones financieras ecuatorianas. Los resultados del modelo permitieron aceptar las cuatro hipótesis planteadas en este estudio, es decir, que los indicadores financieros seleccionados tienen una afectación en la rentabilidad de las instituciones bancarias ecuatorianas, y este impacto es negativo y positivo de forma significativa según el indicador. Los estudios previos consultados para esta investigación coinciden y refuerzan los resultados de este manuscrito.

Los resultados obtenidos implican que la rentabilidad disminuye cuando las variables independientes de liquidez y morosidad aumentan, mientras que la rentabilidad aumenta cuando las variables independientes de calidad de activos y apalancamiento también se incrementan. Por lo tanto, se recomienda revisar continuamente las políticas relacionadas a la recuperación de cartera, niveles de liquidez y otorgamiento de créditos para controlar el incremento de los indicadores de morosidad y liquidez y mantener proporciones adecuadas y legales en los indicadores de calidad de activos y de apalancamiento, y así se pueda obtener el rendimiento esperado. Además, se sugiere realizar seguimiento al entorno externo del banco, a las condiciones macroeconómicas que pueden afectar estas variables y por consiguiente a la rentabilidad.

La investigación realizada aporta a la literatura financiera existente al ampliar el alcance de la muestra a bancos privados grandes y medianos de Ecuador, ya que en su mayoría estudios previos consideran únicamente a bancos ecuatorianos grandes. Otro punto importante es que este estudio analizó el periodo 2016-2019 entregando una investigación previa a la ocurrencia de la pandemia Covid19, por lo tanto, se recomienda que estudios futuros realicen un análisis posterior a la emergencia sanitaria y se comparen los resultados de los estudios pre y post pandemia. Adicionalmente, pueden incluir una muestra mayor que incluya el par de bancos más representativos de la categoría de bancos pequeños. 


\section{Referencias Bibliográficas}

Asociación de Bancos Privados del Ecuador. (2014). Solidez del sistema bancario ecuatoriano. Asobabanca.

Asociación de Bancos Privados del Ecuador. (2018). Coeficiente de apalancamiento. Revista ASOBANCA, 3-4.

Asociación de Bancos Privados del Ecuador. (2020). Activos por bancos. Evolución de la banca ecuatoriana privada, 18.

Asociación de Bancos Privados del Ecuador. (2020). Datalab Asobanca. https://datalab.asobanca.org.ec/resources/site/terminos/Indicadores.pdf

BBVA. (2021). BBVA. https://www.bbva.mx/educacion-financiera///liquidez.html

Buenaño, E. (2003). Eficiencia-X en la banca ecuatoriana durante el periodo 2000 - 2003. Cuestiones

Económicas. https://www.bce.fin.ec/cuestiones_economicas/images/PDFS/2004/No2/Vol.20-22004EdwinBuenano.pdf

Cabellos, J., \& Naito, M. (2015). Determinantes de las instituciones micro financiera peruanas en el periodo 2006-2013. Universidad del Pacífico.

Chavarin, R. (2015). Morosidad en el pago de créditos y rentabilidad de la banca comercial en México. Revista mexicana de economía y finanzas, 73-85.

Comité de Basilea. (1997). Fortalecimiento de la supervisión bancaria mundial. Denver.

Díaz, R. (2018). Determinantes de la Tasa de Morosidad de la Cartera Bruta de Consumo. Superintendencia de Bancos.

Eslava, R., Chacón, E., \& González, H. (2017). Los indicadores financieros de la banca universal en Venezuela. Revista Gestión y Desarrollo Libre, 58-76.

Gurajati, D. (1988). Basic Econometrics (Second). McGraw Hill.

Gutierrez, A., \& Garrido, C. (1994). Calidad de Activos de la Banca Comercial del Bloque de América del Norte. Transiciones financieras y TLC en Canadá, EU y MX, 195-212.

Heredia, F., \& Torres, H. (2019). Gestión de la morosidad para mejorar la rentabilidad de la caja de ahorro y crédito de José Leonardo Ortiz - 2018. Revista Cientifica Institucional Tzhoecoen, 92 -103.

Jara, G., Sánchez, S., Bucarám, R., \& García, J. (2018). Análisis de indicadores de rentabilidad de la pequeña banca privada en el Ecuador a partir de la dolarización. Compendium, 54-76.

Mendoza, E. (2002). De la crisis financiera ecuatoriana: Causas, consecuencias y soluciones. Revista Jurídica, 45-83.

Moreno, C. (2019). Incidencia de la liquidez en la rentabilidad de la banca privada del Ecuador. Ambato: Universidad Técnica de Ambato.

Redacción de Negocios. (2 de enero de 2010). 28 entidades que quebraron por la crisis de 1998 fueron cerradas. El Comercio. Obtenido de 28 entidades que quebraron por la crisis de 1998 fueron cerradas.: http://www.elcomercio.com/actualidad/28-entidades-quebraroncrisis-1998.html

Ricaurte, C., \& Rosero, C. (2017). Evolución de la cartera de crédito comercial de la banca privada ecuatoriana del 2008 al 2013. http://repositorio.uees.edu.ec/123456789/1435 
Rodrigues, U. (2015). Factores determinantes de la rentabilidad de los bancos en los países del Mercosur: Un enfoque contable. Córdoba: Universidad Nacional de Córdoba.

Rodríguez, A., \& Venegas, F. (2012). Liquidez y apalancamiento de la banca comercial en México. Revista Análisis Económico, 73-96. https://www.researchgate.net/publication/326678560_Liquidez_y_apalancamiento_de_la _banca_comercial_en_Mexico

Superintendencia de Bancos. (2004). CAPITULO I.- De la gestión integral y control de riesgos. En LIBRO I.- Normas generales para las instituciones del sistema financiero (págs. 560 570). $\quad$ https://www.superbancos.gob.ec/bancos/wpcontent/uploads/downloads/2017/06/L1_X_cap_I.pdf

Tenesaca, K., Villanueva, J., Malo, Z., \& Higuerey, Á. (2017). Sistema bancario de Ecuador: Una aproximación a sus indicadores de estabilidad y eficiencia. Revista Publicando UTPL, 255 $-273$.

Torres, A. (2018). Análisis de la evolución de la Liquidez de la Banca Privada Ecuatoriana ante Políticas Públicas, periodo 2012-2016. Guayaquil: Universidad de Guayaquil - Facultad de Ciencias Económicas. http://repositorio.ug.edu.ec/handle/redug/34543

Vargas, G., \& Díaz, M. (2017). Crisis y finanzas sustentables: el papel de la deuda y el apalancamiento. Pistas educativas, 271-286.

Velasco, J. (2017). Análisis del índice de morosidad y su impacto en la rentabilidad del sistema de bancos privados grandes ubicados en la provincia de Imbabura en el periodo 2014 2016. Quito: Universidad Internacional SEK. 